\title{
Boosting Circularly Polarized Luminescence of Organic Conjugated Systems via Twisted Intramolecular Charge Transfer
}

\author{
Junfeng Li, ${ }^{1}$ Chenxi Hou, ${ }^{1}$ Chao Huang, ${ }^{1}$ Shanqi Xu, ${ }^{1}$ Xuelei Peng, ${ }^{1}$ Qi Qi, ${ }^{2}$ \\ Wen-Yong Lai $\mathbb{1}^{1,3}$ and Wei Huang ${ }^{1,3}$ \\ ${ }^{1}$ Key Laboratory for Organic Electronics and Information Displays, Institute of Advanced Materials (IAM), Nanjing University of \\ Posts and Telecommunications, 9 Wenyuan Road, Nanjing 210023, China \\ ${ }^{2}$ School of Chemistry and Chemical Engineering, Southeast University, Nanjing 211189, China \\ ${ }^{3}$ Shaanxi Institute of Flexible Electronics (SIFE), Northwestern Polytechnical University (NPU), 127 West Youyi Road, Xi'an, \\ 710072 Shaanxi, China
}

Correspondence should be addressed to Wen-Yong Lai; iamwylai@njupt.edu.cn

Received 30 January 2020; Accepted 22 March 2020; Published 22 April 2020

Copyright (c) 2020 Junfeng Li et al. Exclusive Licensee Science and Technology Review Publishing House. Distributed under a Creative Commons Attribution License (CC BY 4.0).

\begin{abstract}
Realizing a high luminescence dissymmetry factor $\left(g_{\text {lum }}\right)$ is a paramount yet challenging issue in the research field of circularly polarized luminescence (CPL). Here, we reported a novel set of organic conjugated systems with twisted intramolecular charge transfer (TICT) characteristics based on conjugated $o$-carborane-binaphthyl dyads composing of binaphthyl units as chiral electron donors and $o$-carborane units as achiral electron acceptors, demonstrating intense CPL with large $g_{\text {lum }}$ values. Interestingly, single-crystalline $o$ - 1 exhibited a high-level brightness and a large $g_{\text {lum }}$ factor as high as +0.13 , whereas singlecrystalline $o-2$ processed a relatively low brightness with a decreased $g_{\text {lum }}$ value to -0.04 . The significant diversity of CPL-active properties was triggered by the selective introduction of $o$-carborane units onto the binaphthyl units. Benefiting from the large magnetic dipole transition moments in TICT states, the CPL activity of TICT $o$-carborane-based materials exhibited amplified circular polarization. This study provides an efficient molecular engineering strategy for the rational design and development of highly efficient CPL-active materials.
\end{abstract}

\section{Introduction}

In recent years, organic $\pi$-conjugated functional materials [1-3], especially chiroptical materials featuring with circularly polarized luminescence $(\mathrm{CPL})$, have attracted growing interest for their wide potential applications in threedimensional (3D) optical displays [4], optical storage and processing systems [5], color-image projection [6], liquid crystal lasers [7], biological probes and signatures [8], security tags [9], light-emitting diodes [10-12], and, especially, backlighting liquid crystal displays [13]. Concerning CPLactive materials, one of the major targets is to achieve a large luminescence dissymmetry factor $\left(g_{\text {lum }}\right)$, which reflects the level of CPL properties. Generally, $g_{\text {lum }}=2\left(I_{\mathrm{L}}-I_{\mathrm{R}}\right) /\left(I_{\mathrm{L}}+I_{\mathrm{R}}\right)$, in which $I_{\mathrm{L}}$ and $I_{\mathrm{R}}$ denote the intensity of left and right circularly polarized light, respectively [14]. Theoretically, $g_{\text {lum }}$ is simply approximated by $4|m| \mu \mid \cos \theta /\left(|m|^{2}+|\mu|^{2}\right)$, in which $m$ and $\mu$ denote the magnetic and electric transition dipole moments, respectively, and $\theta$ denotes the angle between $m$ and $\mu$ [15]. High $g_{\text {lum }}$ values could only arise from $m$ -allowed and $\mu$-forbidden transitions, while low values are generally induced by $m$-forbidden and $\mu$-allowed transitions. However, owing to the essential large $|\mu|$ and negligible $|m|$ in chiral organic materials, it is generally very challenging to achieve highly efficient CPL activity. The development of a novel organic system featuring with relatively large $|m|$ and depressed $|\mu|$ would contribute to enhancing CPL with large $g_{\text {lum }}$.

To amplify $g_{\text {lum }}$ values, various organic material systems and approaches have been explored by constructing aggregation-induced CPL material systems [16], selfassembly supramolecular material systems [17], Förster resonance energy transfer systems $[18,19]$, or triplet-triplet annihilation upconversion CPL systems [20], etc. Nevertheless, the $\left|g_{\text {lum }}\right|$ values of the existing organic systems are still quite low and generally fall into the range of $10^{-4}$ to $10^{-2}$, which 
hampers largely the further investigation of organic materials for CPL applications [21]. On the other hand, it has been well demonstrated that charge-transfer (CT) organic materials consisting of $\pi$-electron-rich donors and $\pi$-electron-deficient acceptors are endowed with a forbidden electron dipole transition moment and a relatively large magnetic transition moment [22]. It is surmised that intense CPL with large $g_{\text {lum }}$ could thus be achieved for the CT-active materials that are emissive with inherent chirality, which remains yet to be attempted by far.

In this contribution, to verify the above hypothesis, a novel set of organic conjugated systems based on isomeric $o$-carborane-functionalized binaphthyl (BINOL) dyads with the same $(R)$-axial chirality have been designed, synthesized, and investigated, in which $o$-carborane units act as achiral electron acceptors and BINOL units as chiral electron donors. The chemical structures of the resulting $o$-carborane-binaphthyl dyads, 6,6 ${ }^{\prime}$-carborane-substituted BINOL $(o-1)$, and $3,3^{\prime}$-carborane-substituted BINOL (o-2), are depicted in Figure 1(a). The dihedral angles of BINOL are completely dependent on the substituents. The electrondeficient $o$-carborane units are prone to induce molecular charge transfer, leading to a charge-separated state [23, 24]. Twisted intramolecular charge transfer (TICT) emissions from $o-1$ to $o-2$ were observed, presumably originating from the rotational movement of $o$-carborane segments. The formation of the TICT state and the emission mechanism [25] are illustrated in Figures 1(b) and 1(c), in comparison with those of the excited-state proton transfer (ESIPT) [26] and excimer emission [27]. It is worthwhile to note that this represents the first example of CPL-active organic conjugated system with TICT characteristics. Moreover, single crystal $o$ - 1 exhibits intense CPL with a $g_{\text {lum }}$ value of +0.13 , which is attributed to the fine-tuning and precise control of dihedral angles of BINOL in the excited states. Substantially, the TICT process of $o$-carborane-based BINOL molecule is beneficial to regulate its dynamic conformations for enhancing magnetic transition dipole moments and forbidding electric transition dipole moments, thus boosting $g_{\text {lum }}$ values.

\section{Results and Discussion}

The synthetic routes to $o-1$ and $o-2$ are depicted in Supplementary Materials. $o-1$ and $o-2$ were synthesized in an average yield about $53 \%$ by Diels-Alder cross-coupling reaction of decaborane with $(R)-2,2^{\prime}$-diethoxy-6,6'-bis(phenylethynyl)-1,1' -binaphthyl and (R)-2,2' -diethoxy-3,3' -bis(phenylethynyl)-1,1' -binaphthyl using $N, N$-dimethylaniline as a Lewis base. The chemical structures of $o-1$ and $o$-2 were identified by ${ }^{1} \mathrm{H} \cdot \mathrm{NMR},{ }^{13} \mathrm{C} \cdot \mathrm{NMR}$, and ${ }^{11} \mathrm{~B} \cdot \mathrm{NMR}$ spectroscopy as well as X-ray crystal data. Both are stable to $\mathrm{H}_{2} \mathrm{O}$, air, and heat in solution and solid states. Thermogravimetric analysis (TGA) was employed to determine the thermal stability of $o$ 1 and $o-2$. As shown in Figure S1, o- 1 and $o-2$ exhibited good thermal stability with decomposition temperatures which started at approximately $417^{\circ} \mathrm{C}$ and $375^{\circ} \mathrm{C}$, respectively, with a weight loss of $5 \%$ under a $\mathrm{N}_{2}$ atmosphere. Figures S2-3 demonstrated that $o-1$ and $o-2$ showed typical $\pi-\pi^{*}$ bands of the BINOL moieties in the range of 295 to $380 \mathrm{~nm}$, devoid of effective conjugation between BINOL and the $o$-carborane moieties. Solvent-dependent TICT emissions were observed for $o-1$ and $o-2$, showing dual emissions in pure organic solvents [28]. With the increase of the solvent polarity, the emission bands of $o-1$ and $o-2$ exhibited noteworthy bathochromic shifts and their emission intensities decreased remarkably, indicating that the solvent polarity performed a paramount function in regulating the excited-state electronic conformation [29].

To study the chiroptical properties of $o-1$ and $o-2$, the optical behaviors in aggregated states in the $\mathrm{H}_{2} \mathrm{O}$-THF system were investigated. As shown in Figure S4, the solutionstate emissions of $o-1$ and $o-2$ were hardly observed at the water fraction $\left(f_{\mathrm{w}}\right)$ less than $90 \%$ with no aggregation that occurred. When $f_{\mathrm{w}}$ was up to $90 \%$, the weak emission bands of $o-1$ and $o-2$ centered at $592 \mathrm{~nm}$ and $563 \mathrm{~nm}$ swiftly emerged, respectively. As $f_{\mathrm{w}}$ was $95 \%$, the $\lambda_{\mathrm{em}}$ value of $o-1$ had an obvious blue shift by $31 \mathrm{~nm}$ with respect to $f_{\mathrm{w}}$ of $90 \%$, while $o-2$ was only slightly blue shifted by $9 \mathrm{~nm}$ [28]. It was clearly demonstrated that the red-shifted spectra were attributed to the lowest TICT excited states [30]. On the other hand, the selective introduction of $o$ carborane units onto the BINOL skeletons also exerted influence on their aggregated patterns, resulting in different aggregation-induced emission- (AIE-) active properties. In order to explore the possible expression of chirality in aggregates and to obtain more chiral conformations of the assemblies in the ground states, circular dichroism (CD) spectra of $o-1$ and $o-2$ were performed in $\mathrm{H}_{2} \mathrm{O} / \mathrm{THF}$ solutions. CD bands of $o-1$ and $o-2$ displayed an almost mirror image relationship. As depicted in Figure 2 and S5, $o-1$ and $o-2$ exhibited opposite signs in the first Cotton CD band at the position of the $\pi-\pi^{\otimes}$ band, suggesting that the bisignate Cotton effects came originally from the excitation couplings between obliquely oriented neighboring transition dipole moments [31]. In this regard, upon increasing the water fraction, the signal of CD has an obvious decrease and red shift, demonstrating a weak asymmetric nature of the self-assembly architecture of $o-1$. However, the CD signal of $o$-2 did not have an obvious change with the increasing water fraction in the $\mathrm{H}_{2} \mathrm{O} / \mathrm{THF}$ solution. The major causes are that three-dimensional $o$ carborane substituents onto the BINOL skeletons lead to chirality inversion relative to BINOL. In this process, the conformation of $o-2$ underwent a portion of racemization. The positive CD couplet signified that $o-1$ had a predominantly $P$-chiral organization, while negative CD couplet of $o$-2 presented an $M$-chiral organization [32]. It proved that the chiral configuration of $o-2$ underwent a complete inversion relative to that of $o-1$ due to the selective introduction of three-dimensional $o$-carborane substituents onto the BINOL units. These results correlated well with the results from the UV spectra.

To quantitatively access the level of CPL, the degree of CPL is evaluated using the dimensionless Kuhn's anisotropy factor $\left(g_{\text {lum }}\right)$ in photoexcited states. CPL signals of $o-1$ and $o-2$ were measured when the water fraction was $95 \%$ in the $\mathrm{H}_{2} \mathrm{O} / \mathrm{THF}$ solution. The CPL signal is sensitive to external 

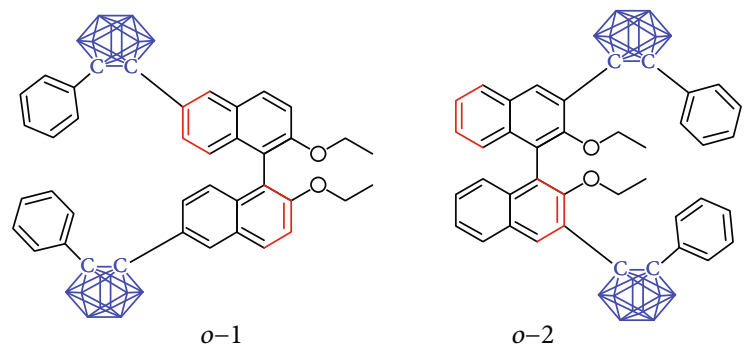

(a)

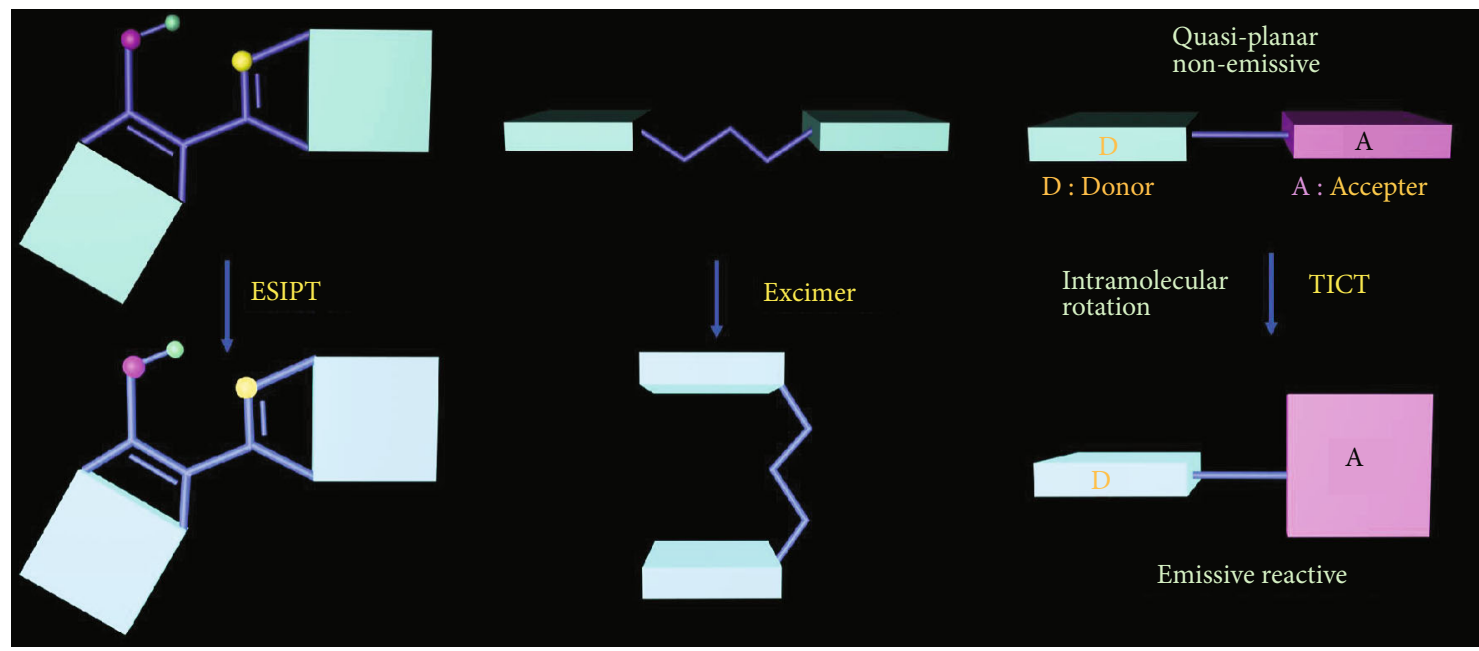

(b)
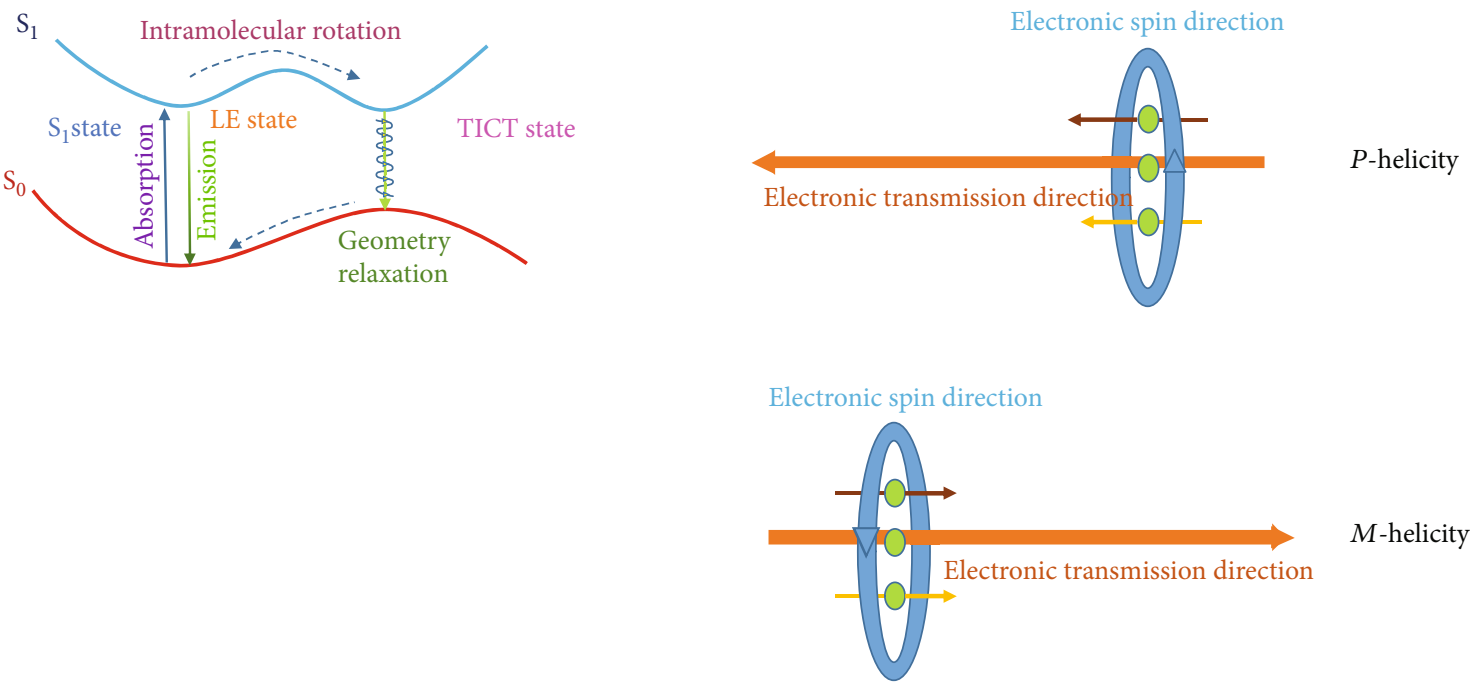

M-helicity

(c)

(d)

FIgure 1: (a) Chemical structures of $o-1$ and $o-2$. (b, c) Schematic illustration of the twisted intramolecular charge transfer (TICT) mechanism, in comparison with those of the excited-state proton transfer (ESIPT) and excimer emission; "D" and "A" refer to electrondonating and electron-accepting units, respectively. (d) The relationship between electronic spin direction and molecular helical organization.

stimuli and also regarded as a remarkable indicator of excited molecular structures. As depicted in Figure 3, the $g_{\text {lum }}$ value for $o-1$ is $+9.13 \times 10^{-4}$ at $561 \mathrm{~nm}$ and for $o-2$ is $-1.81 \times 10^{-3}$ at $627 \mathrm{~nm}$, when $f_{\mathrm{w}}$ is up to $95 \%$. Interestingly, $o-1$ and $o-2$ are almost mirror images, and the signs of the CPL spectra are reverse even though $o-1$ and $o$-2 have the same axially chi- ral BINOL unit. Indeed, the sign of the CPL spectra for $0-1$ is positive with a $P$-chiral organization, whereas that of $o-2$ is negative with a $M$-chiral organization. However, the detected absolute $g_{\text {lum }}$ value decreased by one order of magnitude of $o$ - 1 compared to that of $o-2$, depending on the nature of the excited structures. One reason is that the irregular aggregated 

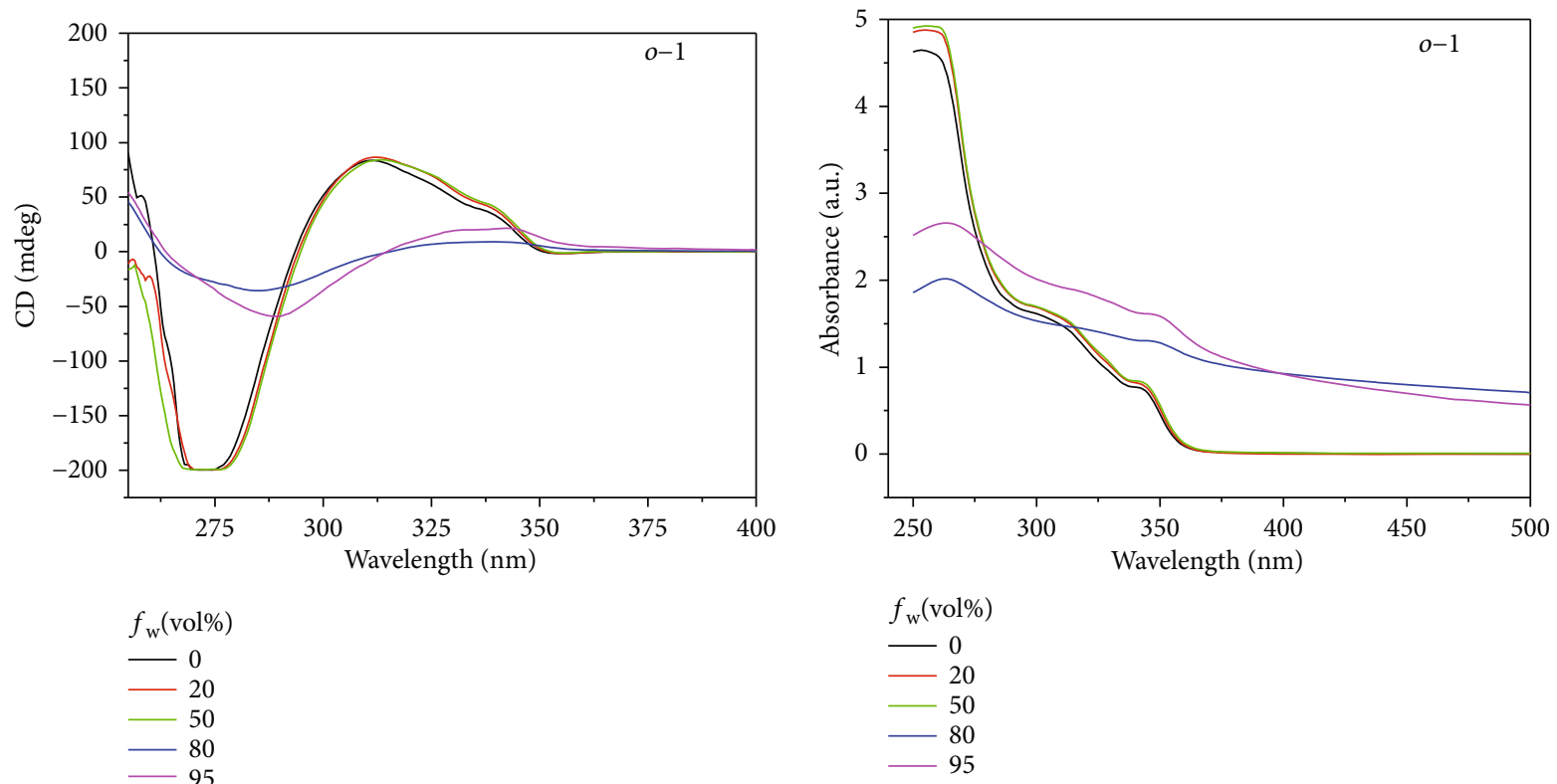

(a)

(b)

Figure 2: (a) CD and (b) UV-Vis spectra of $o-1$ in pure THF and $\mathrm{H}_{2} \mathrm{O} / \mathrm{THF}$ solutions $(v / v, 20: 80,50: 50,20: 80$, and $95: 5)$. Solution concentration: $100 \mu \mathrm{M}$.

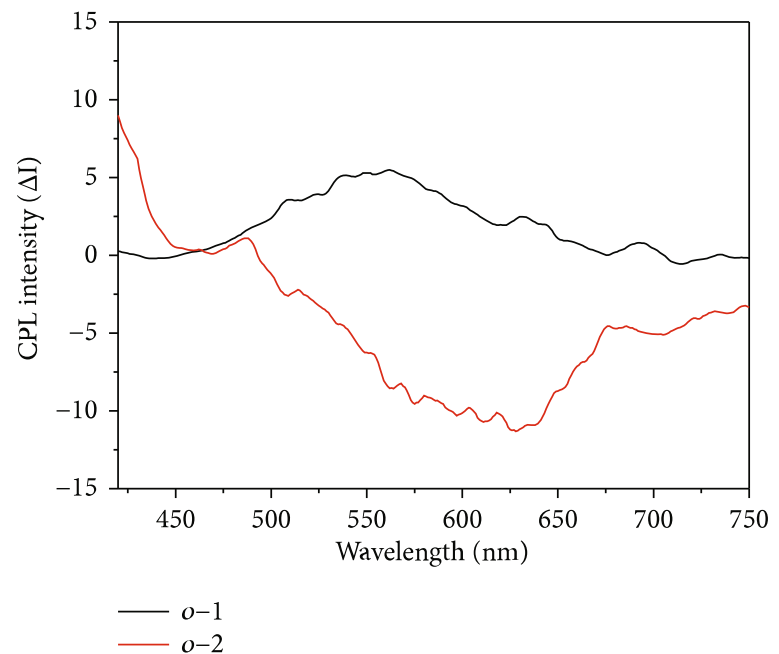

(a)

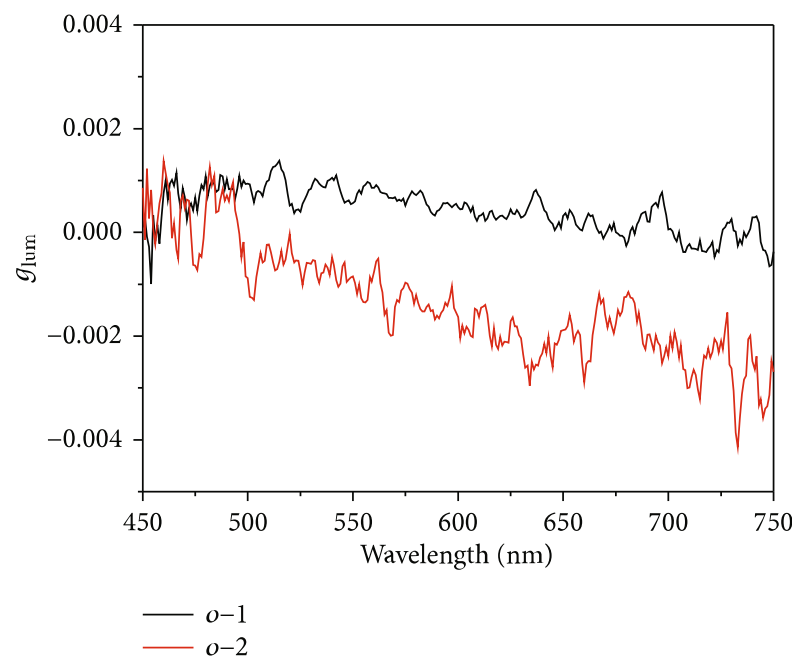

(b)

FIGURE 3: CPL and $g_{\text {lum }}$ spectra of $o-1$ and $o-2$ in $\mathrm{H}_{2} \mathrm{O} / \mathrm{THF}$ solution $(v / v, 95: 5)$. Solution concentration: $10 \mu \mathrm{M}$.

assembles are unfavorable for enhancing the $g_{\text {lum }}$ values, as supported by scanning electron microscopy (SEM) images (Figure S7) [33]. It should be noted that it is readily available to record the CPL signals of $o-1$ and $o-2$ in the self-assemble states, and the detected CPL signals are mainly ascribed to the TICT emissions in the excited states. A dynamic excited molecular structure opens a way for regulating its magnetic and electric transition dipole moments, which is beneficial to boost the $g_{\text {lum }}$ values. This can explain why the $g_{\text {lum }}$ value for $o-2$ was larger than that of $o-1$ in the aggregated states.

To unravel the essence of the CPL sign reversal, the timedependent density functional theory (TD-DFT) with the CAM-B3LYP functional was carried out. CD spectra are dominated by dihedral angles of the BINOL units through the investigation of chiroptical CD signs in the fluidic solution and solid states of BINOL luminophores with the same axial chirality. However, the specific relationship between CPL activity of TICT dyes and their excited conformations has been rarely reported even in aggregated states. While the optimized excited-state $\left(S_{1}\right)$ conformation of $o$-1 is characterized by a highly twisted BINOL core with a twisting angle $(\theta)$ of $-54.20^{\circ}, o-2$ is found to be more planar, featuring a larger $\theta$ of $-129.19^{\circ}$ (Figure 4). It can be inferred that the conformation of $o-2$ underwent a reversion with respect to $o-1$ even though both compounds have the same axially chiral BINOL units [34]. The $g_{\text {lum }}$ value of $o-2$ is larger than 


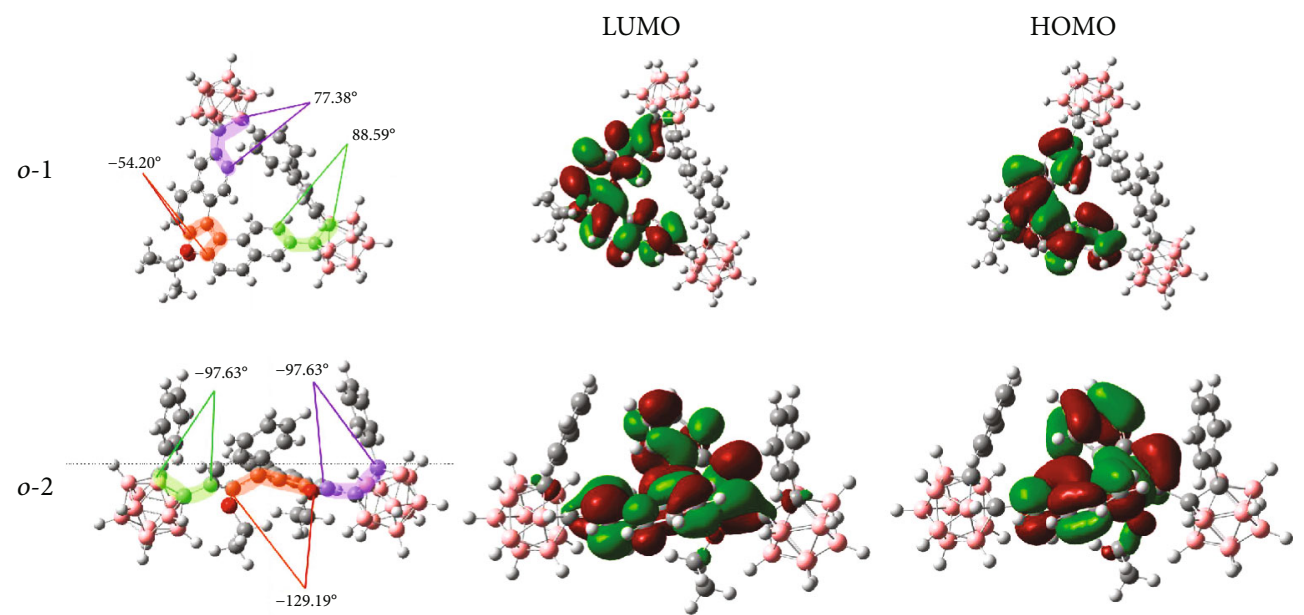

FIGURE 4: Optimized geometries and their frontier orbitals for $\mathrm{S}_{1}$ in $o-1$ and $o-2$ using the state-specific polarizable continuum model (PCM) at the TD-DFT CAM-B3LYP/6-31+G(d,p) level in the aggregated states.

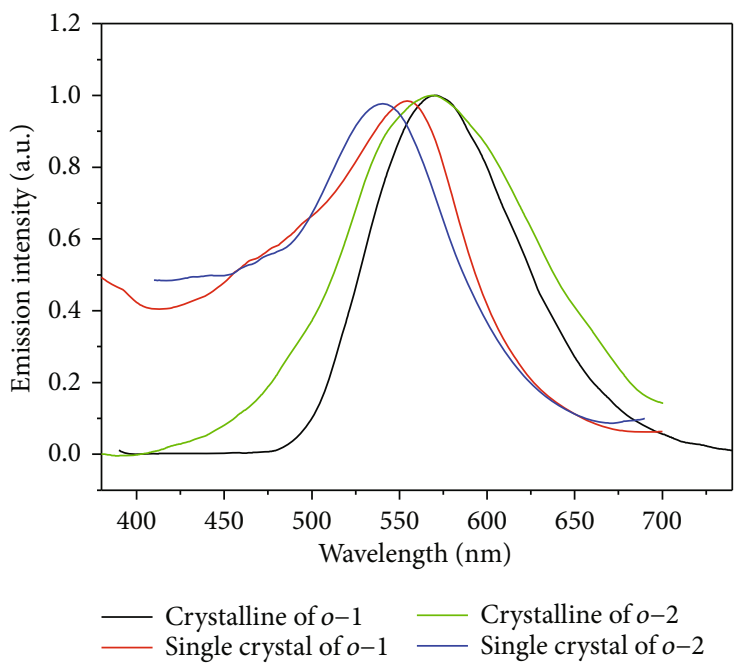

(a)

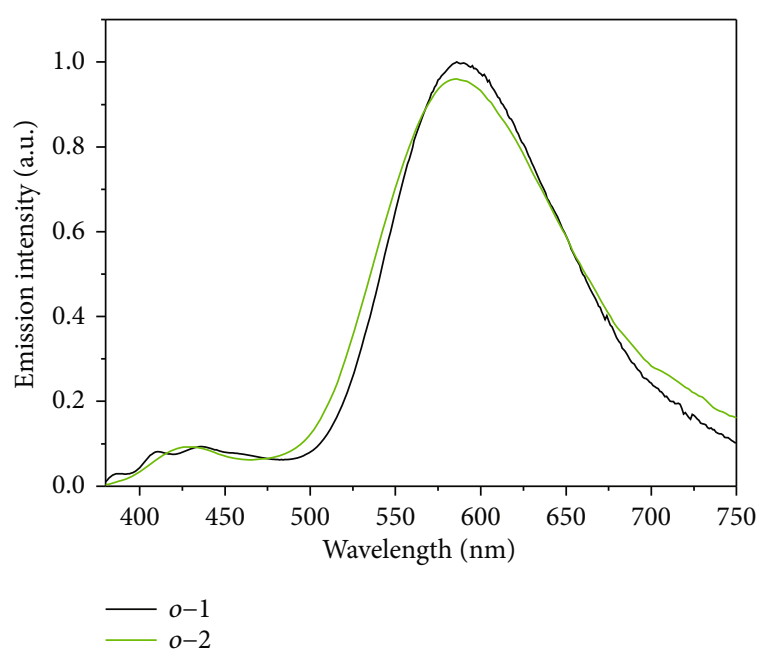

(b)

Figure 5: (a) Normalized emission spectra of $o-1$ and $o-2$ in the single-crystalline states and in the pristine-crystalline states at room temperature. (b) Normalized emission spectra of $o-1$ and $o-2$ in the crystalline states at $77 \mathrm{~K}$.

that of $o-1$ when $f_{\mathrm{w}}$ is $95 \%$, revealing that the $g_{\text {lum }}$ value is mainly determined by the aggregated electronic structure in the excited states. The highest occupied molecular orbital (HOMO) levels in both conformers are located almost on BINOL moieties, while the lowest unoccupied molecular orbital (LUMO) levels reside on BINOL moiety and the connected partial $\mathrm{C}-\mathrm{C}$ bond in one $o$-carborane moiety, giving rise to a piece of direct evidence for the notable characteristic ICT behaviors. Hence, CPL caused by aggregated behaviors comes originally from the TICT states.

The emission features of $o-1$ and $o-2$ crystallines are analyzed by photophysical spectroscopy and low-temperature emission spectra. As depicted in Figure 5(a), crystal powders of $o-1$ and $o-2$ exhibited a strong yellow emission with $\lambda_{\max }$ at $570 \mathrm{~nm}$ and $568 \mathrm{~nm}$ and a high $\Phi_{\mathrm{EM}}$ of $85 \%$ and $78 \%$, respectively, arising from the intrinsic TICT transitions from BINOL units to the $\mathrm{C}-\mathrm{C}$ bond of $o$-carborane moieties. The emission band of single crystal $o-1$ exhibited a $16 \mathrm{~nm}$ hypochromatic shift from $570 \mathrm{~nm}$ to $554 \mathrm{~nm}$, while $o$-2 showed a $29 \mathrm{~nm}$ hypochromatic shift from $568 \mathrm{~nm}$ to $539 \mathrm{~nm}$. The results indicated that $o-1$ and $o-2$ possessed not only AIE but also crystallization-induced emission (CIE) properties due to the dynamic rotational movement of $o$-carborane units $[35,36]$. To verify this assumption, the fluorescence spectra of $o-1$ and $o-2$ at $77 \mathrm{~K}$ were recorded in the crystalline states. Generally, the rotation of $o$-carborane is suppressed in frozen media resulting in LE emission. As shown in Figure 5(b), the new emission bands $o-1$ and $o-2$ were observed in the range of $380-480 \mathrm{~nm}$ with the vibrational peaks, which were attributed to the emission from the localized excited (LE) states. The other broadband was attributed to the TICT emissions at $547 \mathrm{~nm}$ [37], indicating that intramolecular rotations take place even in the crystalline states. As a result, solid-state emissions of $o-1$ and $o-2$ were obtained 


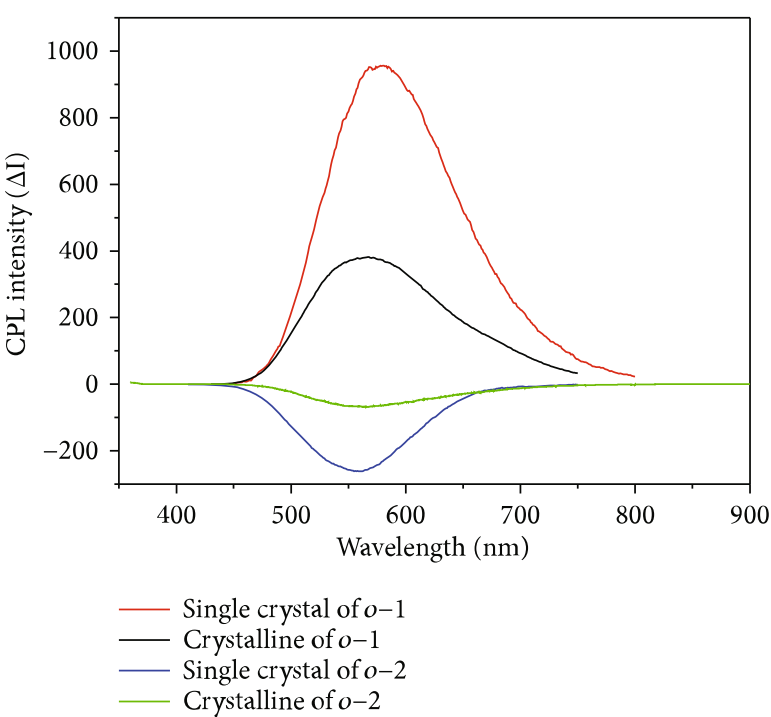

(a)

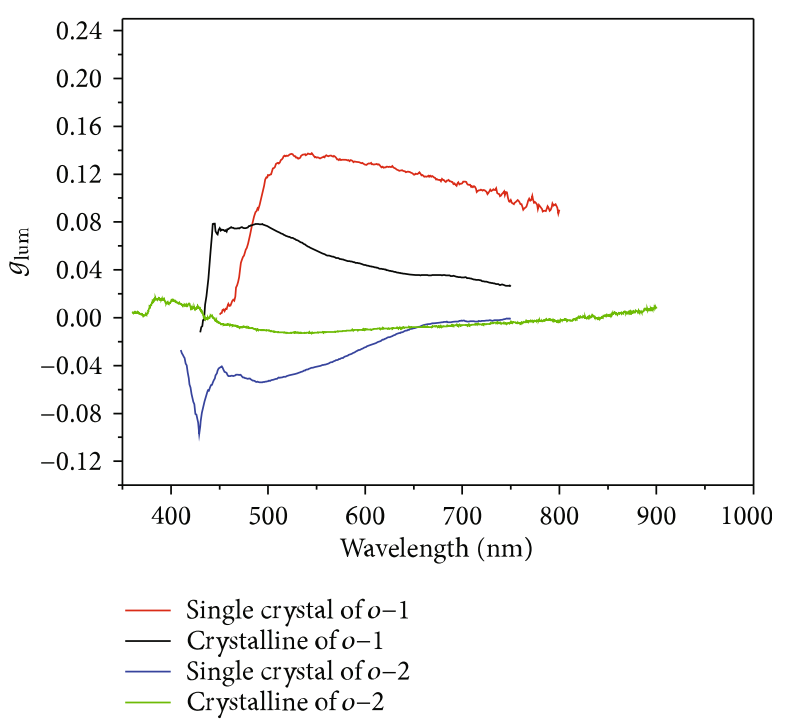

(b)

Figure 6: (a) CPL. (b) $g_{\text {lum }}$ spectra of $o-1$ and $o$-2 in the single-crystalline states and in the pristine-crystalline states at room temperature.

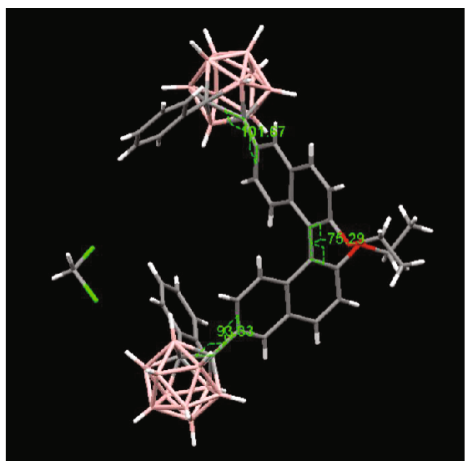

(a)

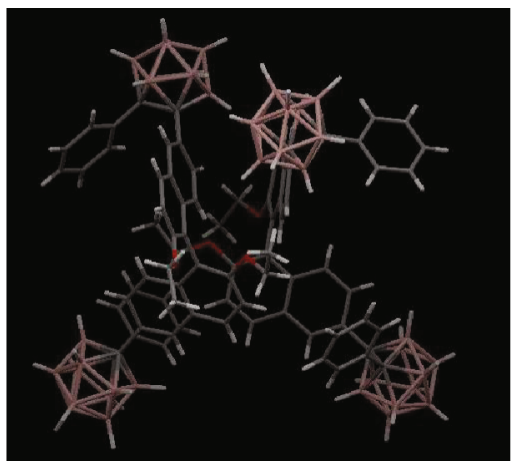

(b)

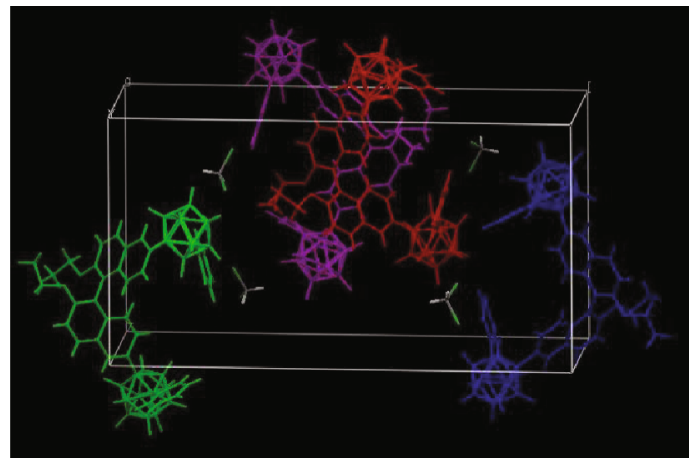

(c)

Figure 7: (a) ORTEP drawing, (b) intermolecular interactions, and (c) molecular packing of $o-1$.

via TICT electronic transitions, originating from unidirectional movement of $o$-carborane moieties in the crystalline states.

As depicted in Figure S6, CD spectra of $o-1$ in the KBrdispersed states displayed a bisignate Cotton effect with a positive wave at around $322 \mathrm{~nm}$ accompanied by a pronounced wavelength at around $360 \mathrm{~nm}$ and a negative wave at around $279 \mathrm{~nm}$, indicating that chirality signal transferred from BINOL to $o$-carborane units. Meanwhile, a well-resolved bisignate CD signal of $o-2$ was observed with a positive wave at around $310 \mathrm{~nm}$ accompanied by a pronounced wavelength at around $247 \mathrm{~nm}$ and a negative wave at around $265 \mathrm{~nm}$. These results also correlated well with the results of the UV spectra. The measurements of the solid-state CPL of $o-1$ and $o-2$ were carried out. As shown in Figure 6, $o-1$ and $o-2$ in crystal powders exhibit strong CPL emissions located at $563 \mathrm{~nm}$ and $562 \mathrm{~nm}$, and their $g_{\text {lum }}$ values are +0.05 and -0.01 , respectively. Also, it is notable that, in single-crystalline states, the CPL emission bands of $o-1$ and $o-2$ centered at $578 \mathrm{~nm}$ and $556 \mathrm{~nm}$, and their $g_{\text {lum }}$ values are +0.13 and -0.04 , respectively. The CPL signs agreed with those of the CD signals at the longest wavelengths, indicating that the ground and excited states exhibited similar conformations. Thus, the chirality signals of $o-1$ and $o-2$ inherently originated from $P$-chiral organization and $M$-chiral organization, respectively. The distinct difference of $g_{\text {lum }}$ further confirms that $o-1$ may take a more twisted conformation in the excited states.

To better understand the CPL properties of $o-1$ and $o-2$, crystals were obtained from the mixed solutions of methanol and dichloromethane. Their single-crystal structures were investigated in detail, and crystallographic data are given in Table S1-2. Both $o-1$ and $o-2$ adopted a three-dimensional twisted conformational structure in orthorhombic $\mathrm{P} 2{ }_{1} 2_{1} 2_{1}$. The data are summarized in Table S1-2. The dihedral angles between BINOL and the $\mathrm{C}-\mathrm{C}$ bonds in the $o$ carborane units of $o-1$ are $+106.67^{\circ}$ and $+93.33^{\circ}$, respectively. The BINOL angle is $-75.29^{\circ}$. As shown in Figures 7 and 8 , no distinct $\pi-\pi$ stacking between BINOL units was found in the crystalline packing for $o-1$ and $0-2$, which facilitated the rotation of the $o$-carborane cluster to induce transformations among various conformation states. 


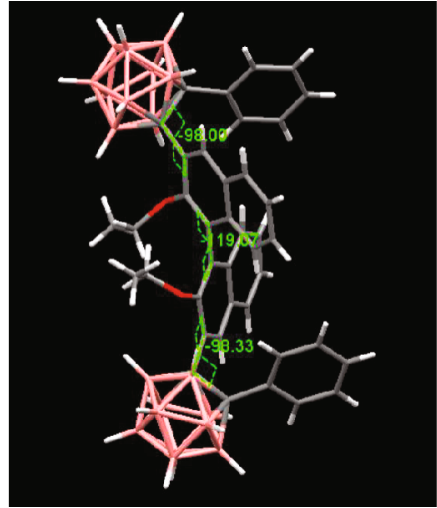

(a)

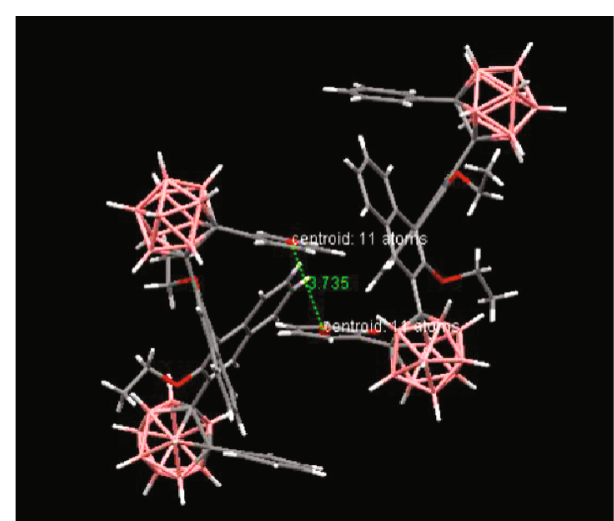

(b)

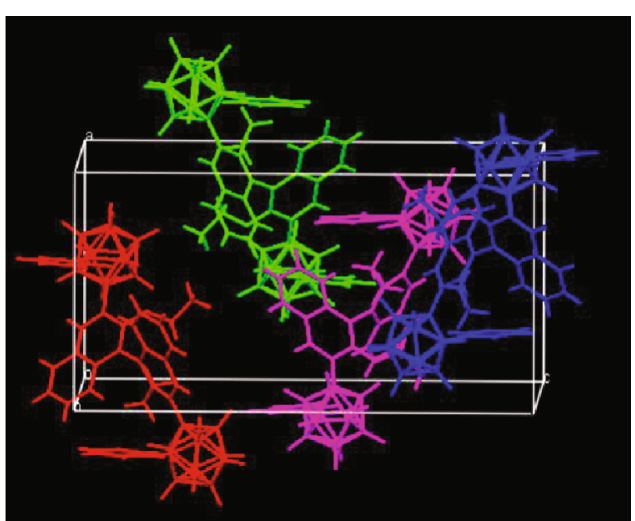

(c)

FIGURE 8: (a) ORTEP drawing, (b) intermolecular interactions, and (c) molecular packing of $o-2$.

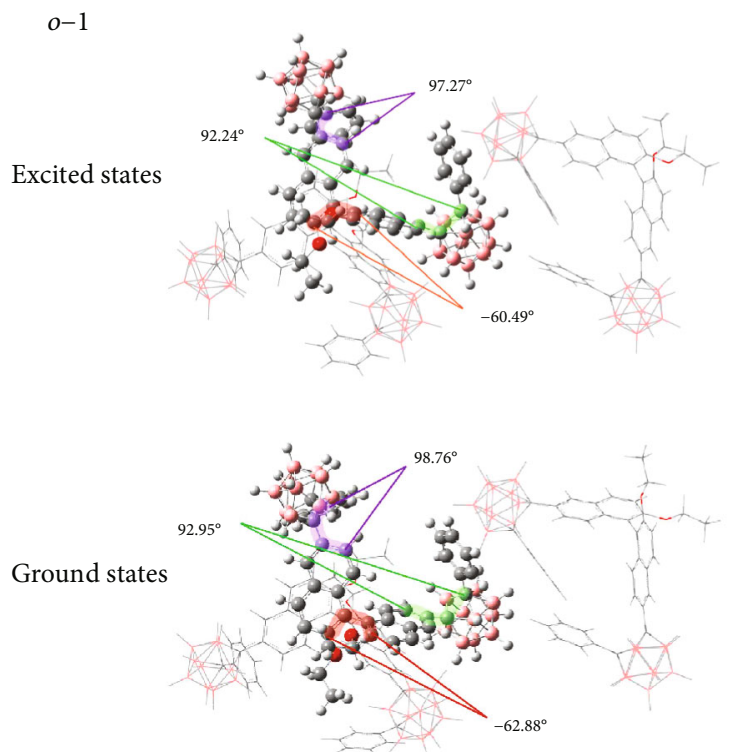

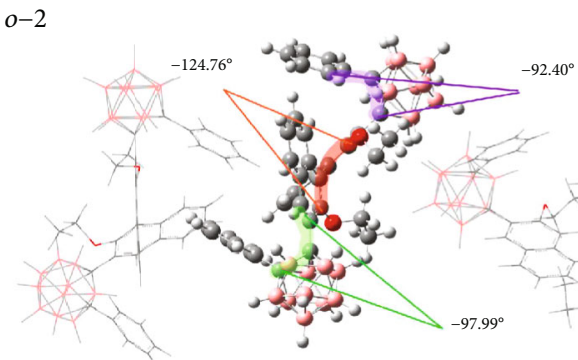

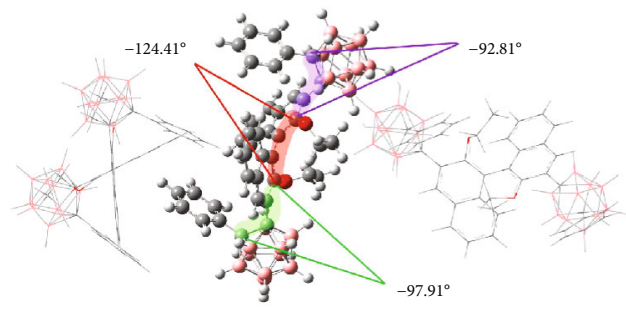

Figure 9: Optimized geometries for $S_{0}$ and $S_{1}$ of $o-1$ and $o-2$ using the state-specific PCM at the TD-DFT CAM-B3LYP/6-31+G(d,p) level in the crystalline states.

That is, the crystallization of $o-1$ takes a more twisted conformation than that of the aggregated states, which thus induces the TICT activity upon crystallization. The TICT states are responsible for CPL in the crystalline states, suggesting that the chirality signals transferred from BINOL to $o$-carborane units through space energy transfer [38]. As shown in Figure 8, the dihedral angles of BINOL in 0 - 2 was $-119.07^{\circ}$, meaning that intramolecular steric repulsion of $o-2$ was stronger than that of $o-1$. The CD intensities at the longest wavelengths are significantly affected by the dihedral angles. This explains why $o-1$ and $o-2$ show the same axial chirality but exhibit opposite signs in $\mathrm{CD}$ and $\mathrm{CPL}$.

TD-DFT with the CAM-B3LYP functional of $o-1$ and $o-2$ in the crystalline states was also carried out to better understand the CPL properties. CD and CPL spectra are closely related to the dihedral angles of BINOL in ground states. As shown in Figure 9, the crystalline-phase calculations with the finite-size cluster model based on the single-crystal data predicted that the dihedral angles $\theta((\mathrm{O}) \mathrm{C}-\mathrm{C}-\mathrm{C}-\mathrm{C}(\mathrm{O}))$ of BINOL are obviously different $\left(-62.88^{\circ}\right.$ for $o-1,-124.41^{\circ}$ for $o$-2), taking reversible signs in $\mathrm{CD}$, whereas the dihedral angles $\theta((\mathrm{O}) \mathrm{C}-\mathrm{C}-\mathrm{C}-\mathrm{C}(\mathrm{O}))$ of $\mathrm{BINOL}$ are $-60.49^{\circ}$ and $-124.76^{\circ}$ for $o-1$ and $o-2$ in the excited states, respectively. Dihedral angles of BINOL units for $o-1$ and $o-2$ are dynamically regulated by the excitation, and the values of the angle change are $2.39^{\circ}$ for $o-1$ and $-0.35^{\circ}$ for $o-2$. The results demonstrated that $o-1$ underwent a large dynamic conformational change upon $S_{0} \longrightarrow S_{1}$ excitation in the crystalline states. However, the conformational transitions upon $S_{0} \longrightarrow S_{1}$ excitation of $o-2$ were substantially suppressed in the crystalline phase. To put it simple, $o-1$ adopted a more twisted conformation than that of $o$-2, driven by the bulky substituents attached onto the open-type BINOL. There is no denying, though, that the detected larger $g_{\text {lum }}$ value of $o$ 1 in the crystalline state is ascribed to subtle variations in the excited-state conformations, resulting in an amplification of magnetic transition dipole moment, whereas the 
conformation of $o$-2 was almost not altering, leading to no alteration of the magnetic and electric transition dipole moments. Natural transition orbital (NTO) is employed to analyze a $\pi-\pi^{*}$ transition of the BINOL moiety and additional TICT contribution from the central BINOL donor to the peripheral $o$-carborane acceptor for $o-1$ and $o-2$, as shown in Figure S10 [39]. Hence, the nature of the CPL for $o-1$ and $o-2$ is the result of TICT states in the crystalline phases. Theoretically, $g_{\text {lum }}$ is defined as $4 \mid$ $m|\mu| \cos \theta /\left(|m|^{2}+|\mu|^{2}\right)$. Accordingly, high $g_{\text {lum }}$ values could only be achieved for $m$-allowed and $\mu$-forbidden transitions, while low values are generally expected for $m$ -forbidden and $\mu$-allowed transitions. To deduce the origin of these anomalous $g_{\text {lum }}$ values, we performed TD-DFT analysis at the TD-TPSSTPSS/6-31G(d,p) level [40]. The magnetic and electric transition dipole moments from $S_{0} \longrightarrow S_{1}$ state for $o-1$ were 0.063 and 0.068 , respectively, and for $o$-2 were 0.144 and 0.645 , respectively. Meanwhile, the two transition dipole moments from $S_{1} \longrightarrow$ $S_{0}$ state, $m$ and $\mu$, for $o$ - 1 (i.e., with an angle of $75.51^{\circ}$ ) were 0.800 and 1.002 , respectively, and for $o$-2 (i.e., with an angle of $176.10^{\circ}$ ) were 0.153 and 1.887 , respectively. These data showed that $o-1$ possessed an anomalously magnetic transition dipole moment in the $S_{1} \longrightarrow S_{0}$ state. The $g_{\text {lum }}$ value is derived as a combination of $|m|$ and $|\mu|$ in a relationship of $g_{\text {lum }}$ by $(|m| /|\mu| \cos \theta)$. Thus, unlike conventional organic molecules with negligible magnetic transition dipole moment contributions, it stands to reason that the choice of $3,3^{\prime}$ - and $6,6^{\prime}$-substituted $o$-carborane in $o$ 1 and $o$-2 exerts a great influence on the molecular packing and electronic transmission, responsible for modulating the magnetic and electric transition dipole moments. The dynamic rotational movements of $o$-carborane moieties may lead to a small $|\mu|$ value and enhance the corresponding $|m|$ value, arising an enhancement of the $g_{\text {lum }}$ value [41]. This is why $o$ - 1 manifests a larger $g_{\text {lum }}$ than that of $o-2$ in crystalline states [42]. Moreover, the choice of $3,3^{\prime}-$ and $6,6^{\prime}$-substituted $o$-carborane in $o-1$ and $o-2$ can regulate their arrangement of dipoles in the crystalline and even resolve their electronic spin directions upon photoexcited states, which have a direct correction with their chirality organizations, as shown in Figure $1(\mathrm{~d})$. This gives a direct explanation that $o-1$ and $o-2$ exhibit inverse signals.

\section{Conclusions}

To conclude, a set of CPL-active organic conjugated systems based on chiral $o$-carborane-binaphthyl dyads have been designed, synthesized, and systematically investigated. Both dyads, in the crystalline states, exhibited TICT emissions owing to the dynamic rotational movement of $o$-carborane units, which represents the first example of CPL-active organic material system with TICT characteristics. Moreover, single-crystal $o$ - 1 exhibits intense CPL with a boosting $g_{\text {lum }}$ value approaching +0.13 , the highest value ever recorded in organic conjugated systems by far. Controlling dihedral angles in BINOL units is responsible for regulating molecular conformations, and the electron-deficient $o$-car- borane is beneficial to enhance the magnetic transition dipole moments and forbidding the electric transition dipole moments, thus promoting a larger $g_{\text {lum }}$ value. This study provides an efficient molecular engineering strategy for the rational design and development of highly efficient CPLactive materials with boosting $g_{\text {lum }}$ values.

\section{Appendix}

The first example of circularly polarized luminescence(CPL-) active organic conjugated system with twisted intramolecular charge transfer (TICT) characteristics is reported. Impressively, single-crystal $o$ - 1 exhibits intense CPL with a boosting $g_{\text {lum }}$ value approaching +0.13 , the highest value ever recorded in organic conjugated systems by far. The results provide an efficient molecular engineering strategy for the rational design and development of highly efficient CPLactive materials.

\section{Conflicts of Interest}

The authors declare no competing financial interest.

\section{Authors' Contributions}

J. Li, W.-Y. Lai, and W. Huang conceived the idea and designed the experiments, J. Li, C. Hou, C. Huang conducted the experiments, X. Peng performed the X-ray measurements, Q. Qi did the DFT calculations, and J. Li, S. Xu, Q. Qi, and W.-Y. Lai analyzed and discussed the data. J. Li made the draft, W.-Y. Lai revised the manuscript, and all the authors contributed to the writing of the manuscript.

\section{Acknowledgments}

We acknowledge financial support from the National Natural Science Foundation of China (Nos. 21975130, 21835003, 21404059, 21674050, 91833304, and 21422402), the National Basic Research Program of China (973 Program) (Nos. 2017YFB0404501, 2014CB648300), the Six Talent Peaks Project in Jiangsu Province (TD-XCL-009), the 333 Project of Jiangsu Province (BRA2017402), the Leading Talent of Technological Innovation of National Ten-Thousands Talents Program of China, the Excellent Scientific and Technological Innovative Teams of Jiangsu Higher Education Institutions (TJ217038), the Scientific Research Foundation of Nanjing University of Posts and Telecommunications (No. NY219060), the Fundamental Research Funds for the Central Universities, and the open research fund of Key Laboratory of MEMS of Ministry of Education, Southeast University, and the Big Data Center of Southeast University.

\section{Supplementary Materials}

Supplementary materials such as the detailed description of materials used. Synthetic and analytical procedures. Crystal data for $0-1$ and $0-2$. UV-Vis, PL, and NMR spectra are presented. DFT calculation details and additional tables and figures related to this article are provided. X-ray crystallographic data in CIF for CCDC 1914133 for $o-1$ and 
1914144 for $o$-2 can be obtained free of charge from the Cambridge Crystallographic Data Centre via http://ww.ccdc .cam.ac.uk/data_request/cif and from the authors. (Supplementary Materials)

\section{References}

[1] Z. An, C. Zheng, Y. Tao et al., "Stabilizing triplet excited states for ultralong organic phosphorescence," Nature Materials, vol. 14, no. 7, pp. 685-690, 2015.

[2] L. H. Xie, C. R. Yin, W. Y. Lai, Q. L. Fan, and W. Huang, "Polyfluorene-based semiconductors combined with various periodic table elements for organic electronics," Progress in Polymer Science, vol. 37, no. 9, pp. 1192-1264, 2012.

[3] J. Li, X. Peng, C. Huang, Q. Qi, W.-Y. Lai, and W. Huang, "Control of circularly polarized luminescence from a boron ketoiminate-based $\pi$-conjugated polymerviaconformational locks," Polymer Chemistry, vol. 9, no. 43, pp. 5278-5285, 2018.

[4] K. Takaishi, K. Iwachido, R. Takehana, M. Uchiyama, and T. Ema, "Evolving fluorophores into circularly polarized luminophores with a chiral naphthalene tetramer: proposal of excimer chirality rule for circularly polarized luminescence," Journal of the American Chemical Society, vol. 141, no. 15, pp. 6185-6190, 2019.

[5] Y. Yang, R. C. da Costa, M. J. Fuchter, and A. J. Campbell, "Circularly polarized light detection by a chiral organic semiconductor transistor," Nature Photonics, vol. 7, no. 8, pp. 634-638, 2013.

[6] Y. Nagata, K. Takagi, and M. Suginome, "Solid polymer films exhibiting handedness-switchable, full-color-tunable selective reflection of circularly polarized light," Journal of the American Chemical Society, vol. 136, no. 28, pp. 9858-9861, 2014.

[7] C. Mowatt, S. M. Morris, M. H. Song, T. D. Wilkinson, R. H. Friend, and H. J. Coles, "Comparison of the performance of photonic band-edge liquid crystal lasers using different dyes as the gain medium," Journal of Applied Physics, vol. 107, no. 4, article 043101, 2010.

[8] S. Petoud, G. Muller, E. G. Moore et al., "Brilliant Sm, Eu, Tb, and Dy chiral lanthanide complexes with strong circularly polarized luminescence," Journal of the American Chemical Society, vol. 129, no. 1, pp. 77-83, 2007.

[9] R. Carr, N. H. Evans, and D. Parker, "Lanthanide complexes as chiral probes exploiting circularly polarized luminescence," Chemical Society Reviews, vol. 41, no. 23, pp. 7673-7686, 2012.

[10] J. R. Brandt, X. Wang, Y. Yang, A. J. Campbell, and M. J. Fuchter, "Circularly polarized phosphorescent electroluminescence with a high dissymmetry factor from PHOLEDs based on a platinahelicene," Journal of the American Chemical Society, vol. 138, no. 31, pp. 9743-9746, 2016.

[11] M. Li, S. H. Li, D. Zhang et al., "Stable enantiomers displaying thermally activated delayed fluorescence: efficient OLEDs with circularly polarized electroluminescence," Angewandte Chemie International Edition, vol. 57, no. 11, pp. 2889-2893, 2018.

[12] T. Zhao, J. Han, X. Jin et al., "Dual-mode induction of tunable circularly polarized luminescence from chiral metal-organic frameworks," Research, vol. 2020, article 6452123, 12 pages, 2020.

[13] M. Grell and D. D. C. Bradley, "Polarized luminescence from oriented molecular materials," Advanced Materials, vol. 11, no. 11, pp. 895-905, 1999.
[14] K. Takaishi, S. Hinoide, T. Matsumoto, and T. Ema, "Axially Chiralperi-Xanthenoxanthenes as a circularly polarized luminophore," Journal of the American Chemical Society, vol. 141, no. 30, pp. 11852-11857, 2019.

[15] H. Maeda and Y. Bando, "Recent progress in research on stimuli-responsive circularly polarized luminescence based on \&pi;-conjugated molecules," Pure and Applied Chemistry, vol. 85, no. 10, pp. 1967-1978, 2013.

[16] J. Liu, H. Su, L. Meng et al., "What makes efficient circularly polarised luminescence in the condensed phase: aggregationinduced circular dichroism and light emission," Chemical Science, vol. 3, no. 9, pp. 2737-2747, 2012.

[17] Y. Sang, D. Yang, P. Duan, and M. Liu, "Towards homochiral supramolecular entities from achiral molecules by vortex mixing-accompanied self-assembly," Chemical Science, vol. 10, no. 9, pp. 2718-2724, 2019.

[18] E. M. Sánchez-Carnerero, F. Moreno, B. L. Maroto et al., "Circularly polarized luminescence by visible-light absorption in a chiral O-BODIPY dye: unprecedented design of CPL organic molecules from achiral chromophores," Journal of the American Chemical Society, vol. 136, no. 9, pp. 3346-3349, 2014.

[19] D. Yang, P. Duan, L. Zhang, and M. Liu, "Chirality and energy transfer amplified circularly polarized luminescence in composite nanohelix," Nature Communications, vol. 8, no. 1, article 15727, 2017.

[20] J. Han, P. Duan, X. Li, and M. Liu, "Amplification of circularly polarized luminescence through triplet-triplet annihilationbased photon upconversion," Journal of the American Chemical Society, vol. 139, no. 29, pp. 9783-9786, 2017.

[21] G. Park, H. Kim, H. Yang et al., "Amplified circularly polarized phosphorescence from co-assemblies of platinum(ii) complexes,” Chemical Science, vol. 10, no. 5, pp. 1294-1301, 2019.

[22] M. Ziegler and A. von Zelewsky, "Charge-transfer excited state properties of chiral transition metal coordination compounds studied by chiroptical spectroscopy," Coordination Chemistry Reviews, vol. 177, no. 1, pp. 257-300, 1998.

[23] W. Chi, Q. Qiao, R. Lee et al., "A photoexcitation-induced twisted intramolecular charge shuttle," Angewandte Chemie International Edition, vol. 58, no. 21, pp. 7073-7077, 2019.

[24] S. Wang, X. Yan, Z. Cheng, H. Zhang, Y. Liu, and Y. Wang, "Highly efficient near-infrared delayed fluorescence organic light emitting diodes using a phenanthrene-based chargetransfer compound," Angewandte Chemie International Edition, vol. 127, no. 44, pp. 13260-13264, 2015.

[25] Z. R. Grabowski, K. Rotkiewicz, and W. Rettig, "Structural changes accompanying intramolecular electron transfer: focus on twisted intramolecular charge-transfer states and structures," Chemical Reviews, vol. 103, no. 10, pp. 3899-4032, 2003.

[26] C.-C. Hsieh, C.-M. Jiang, and P.-T. Chou, "Recent experimental advances on excited-state intramolecular proton coupled electron transfer reaction," Accounts of Chemical Research, vol. 43, no. 10, pp. 1364-1374, 2010.

[27] H. Saigusa and E. C. Lim, "Excimer formation in van der waals dimers and clusters of aromatic molecules," Accounts of Chemical Research, vol. 29, no. 4, pp. 171-178, 1996.

[28] K. Wee, W. Han, D. W. Cho, S. Kwon, C. Pac, and S. O. Kang, "Carborane photochemistry triggered by aryl substitution: carborane-based dyads with phenyl carbazoles," Angewandte Chemie International Edition, vol. 51, no. 11, pp. 2677-2680, 2012. 
[29] J. Li, C. Yang, X. Peng et al., "Stimuli-responsive solid-state emission fromo-carborane-tetraphenylethene dyads induced by twisted intramolecular charge transfer in the crystalline state," Journal of Materials Chemistry C, vol. 6, no. 1, pp. 1928, 2018.

[30] S. Kim, J. H. Lee, H. So et al., "Spirobifluorene-based $o$-carboranyl compounds: insights into the rotational effect of carborane cages on photoluminescence," Chemistry - A European Journal, vol. 26, no. 2, pp. 548-557, 2020.

[31] F. Freire, J. M. Seco, E. Quiñoá, and R. Riguera, "Chiral amplification and helical-sense tuning by mono- and divalent metals on dynamic helical polymers," Angewandte Chemie International Edition, vol. 50, no. 49, pp. 11692-11696, 2011.

[32] A. Satrijo, S. C. J. Meskers, and T. M. Swager, "Probing a conjugated polymer's transfer of organization-dependent properties from solutions to films," Journal of the American Chemical Society, vol. 128, no. 28, pp. 9030-9031, 2006.

[33] J. Zhang, Q. Liu, W. Wu et al., "Real-time monitoring of hierarchical self-assembly and induction of circularly polarized luminescence from achiral luminogens," ACS Nano, vol. 13, no. 3, pp. 3618-3628, 2019.

[34] R. Aoki, R. Toyoda, J. F. Kögel et al., "Bis(dipyrrinato)zinc(II) complex chiroptical wires: exfoliation into single strands and intensification of circularly polarized luminescence," Journal of the American Chemical Society, vol. 139, no. 45, pp. 16024-16027, 2017.

[35] H. Naito, Y. Morisaki, and Y. Chujo, "o-carborane-based anthracene: a variety of emission behaviors," Angewandte Chemie International Edition, vol. 127, no. 17, pp. 5173-5176, 2015.

[36] H. So, J. H. Kim, J. H. Lee, H. Hwang, D. K. An, and K. M. Lee, "Planarity of terphenyl rings possessing $o$-carborane cages: turning on intramolecular-charge-transfer-based emission," Chemical Communications, vol. 55, no. 96, pp. 14518-14521, 2019.

[37] H. Naito, K. Nishino, Y. Morisaki, K. Tanaka, and Y. Chujo, "Solid-state emission of the anthracene-o-carborane dyad from the twisted-intramolecular charge transfer in the crystalline state," Angewandte Chemie International Edition, vol. 56, no. 1, pp. 254-259, 2017.

[38] K. Takaishi, M. Yasui, and T. Ema, "Binaphthyl-bipyridyl cyclic dyads as a chiroptical switch," Journal of the American Chemical Society, vol. 140, no. 16, pp. 5334-5338, 2018.

[39] F. Gao, R. Du, C. Han et al., "High-efficiency blue thermally activated delayed fluorescence from donor-acceptor-donor systems via the through-space conjugation effect," Chemical Science, vol. 10, no. 21, pp. 5556-5567, 2019.

[40] S. Sato, A. Yoshii, S. Takahashi, S. Furumi, M. Takeuchi, and H. Isobe, "Chiral intertwined spirals and magnetic transition dipole moments dictated by cylinder helicity," Proceedings of the National Academy of Sciences of the United States of America, vol. 114, no. 50, pp. 13097-13101, 2017.

[41] J. L. Alonso-Gómez, J. L. Alonso-Gómez, P. Rivera-Fuentes, N. Harada, N. Berova, and F. Diederich, "An enantiomerically pure alleno-acetylenic macrocycle: synthesis and rationalization of its outstanding chiroptical response," Angewandte Chemie International Edition, vol. 48, no. 30, pp. 5545-5548, 2009.

[42] J. Han, D. Yang, X. Jin, Y. Jiang, M. Liu, and P. Duan, "Enhanced circularly polarized luminescence in emissive charge-transfer complexes," Angewandte Chemie International Edition, vol. 58, no. 21, pp. 7013-7019, 2019. 